\title{
DETERMINAÇÃO DO TEOR DE FENÓLICOS TOTAIS DO Hibiscus sabdariff HIDROETANÓLICA. EMPREGANDO EXTRAÇÃO
}

\author{
G. H. ROSSATTO ${ }^{1}$, M. M. M. BINDES ${ }^{1}$, V. L. CARDOSO ${ }^{1}$ e M. H. M. REIS ${ }^{1}$ \\ ${ }^{1}$ Universidade Federal de Uberlândia, Faculdade de Engenharia Química \\ E-mail para contato: gustavorossatto@hotmail.com
}

\begin{abstract}
RESUMO - O Hibiscus sabdariffa é uma planta rica em compostos bioativos comumente encontrada no Brasil. Este trabalho tem o objetivo determinar a melhor condição de extração de polifenóis em meio hidroalcoólico. As flores secas do hibisco foram trituradas agitadas em diferentes sistemas com variação de concentração (v/v) de etanol em água (25\%, $50 \%$ e $75 \%)$, variação de concentração $(\mathrm{m} / \mathrm{v})$ de hibisco em solvente $\left(10,55\right.$ e $\left.100 \mathrm{~g} \mathrm{~L}^{-1}\right)$ e variação de tempo (30, 75 e $120 \mathrm{~min}$ ). O teor de compostos fenólicos totais foi determinado por método espectrofotométrico. O valor ótimo da concentração de polifenóis (4696 mgEAG $\mathrm{L}^{-1}$ ) foi obtido nas condições tempo $75 \mathrm{~min}$, razão chá-solvente $100 \mathrm{~g} \mathrm{~L}^{-1}$ e porcentagem de etanol $25 \%$.
\end{abstract}

\section{INTRODUÇÃO}

Amplamente conhecida como hibisco, a Hibiscus Sadbariffa é uma planta da família Malvaceae, sendo introduzida no Brasil pelos escravos. Possui um ciclo anual podendo chegar a 1,80 $\mathrm{m}$ de altura, de tonalidade vermelha e com flor em formato de taça é muito cultivada em regiões tropicais (Maciel et al., 2012). A flor é normalmente usada na produção de comidas e bebidas como chás e geleias. O chá de hibisco é muito popular nas Américas, África e sul da Ásia, é encontrado no comércio internacional como ingrediente em infusões de ervas ou em misturas, dando às bebidas a coloração vermelha e seu sabor característico (Mahadevan et al., 2009; Villani et al., 2013; Zhen et al., 2016).

Nesse tipo de planta podem ser encontrados compostos bioativos como fenólicos e flavonoides como quercetina, gossipetina e seus respectivos glicósidos (Linares et al., 2015). Os compostos bioativos possuem propriedades medicinais que atuam como anti-inflamatórios e na prevenção do câncer (Chang et al., 2014; Zhen et al.,2016).

O tipo de solvente, o tempo, a temperatura e a razão entre material e solvente geralmente influenciam na eficiência da extração (Hu et al.,2016). Os métodos de extração mais usuais são a maceração, percolação e a extração com solventes (Melecchi, 2005; Guindani et al.,2014). O etanol é considerado um solvente seguro, não gera resíduos tóxicos, de grau alimentício (Silva et al.,2016). Esse trabalho tem como objetivo quantificar os fenólicos totais do chá de hibisco em meio hidroalcoólico e encontrar a condição ótima de extração de fenóis totais. 


\section{MATERIAIS E MÉTODOS}

\subsection{Materiais e Preparo do chá}

As flores de hibisco foram adquiridas no mercado local em Uberlândia (Minas Gerais). Para se conseguir o tamanho de partícula desejado, as flores foram processadas usando (Processador de Alimentos Mega Star $60 \mathrm{~Hz}, 500 \mathrm{~W}$ ) por $5 \mathrm{~min}$. O material processado foi colocado e agitado manualmente em um conjunto de peneiras (Peneiras Granutest inox 8x2'), para os experimentos foram utilizadas apenas as partículas que passaram pelo mesh 8 $(2,38 \mathrm{~mm})$. As misturas chá-solvente, diferentes razões chá-solvente para um volume de 50 $\mathrm{mL}$ de solvente, de acordo com o planejamento experimental, foram preparadas, colocadas em erlenmeyers e dispostas em um agitador shaker (Stuart) a $120 \mathrm{rpm}$ e temperatura ambiente, aproximadamente $30^{\circ} \mathrm{C}$.

\subsection{Planejamento Experimental}

Para verificar os efeitos de razão chá-solvente, fração de etanol e tempo no rendimento da extração de polifenóis foi feito um planejamento fatorial $3^{\mathrm{k}}$ fracionário $\left(3^{3-1}\right)$ totalizando 9 experimentos. Os fatores estudados tempo $\mathrm{X}_{1}(30,60$ e $120 \mathrm{~min})$, razão chá-solvente $\mathrm{X}_{2}(10$, 55 e $\left.100 \mathrm{~g} \mathrm{~L}^{-1}\right)$ e fração de etanol em água $\mathrm{X}_{3}(25,50$ e $75 \%)$ foram avaliados em três níveis equidistantes $(-1,0,+1)$. Para análise dos resultados foi utilizada a metodologia de superfície de resposta (RSM). O extrato foi filtrado em um filtro de nylon (Medeiros 102).

\subsection{Determinação de Fenólicos Totais}

O teor de fenólicos totais foi determinado pelo reagente Folin Ciocalteu, de acordo com (Kumar et al., 2012). Os tubos de ensaio foram agitados e deixados em local escuro por 60 min e a absorbância das misturas de coloração azul foram medidas em espectrofotômetro (UV - 1240 Shimadzu) a $760 \mathrm{~nm}$. O teor de polifenóis totais foi calculado de acordo com o equivalente em ácido gálico utilizando a curva de calibração preparada a partir da solução padrão.

\section{Resultados e Discussão}

\subsection{Planejamento Experimental}

A Tabela 1 representa o Planejamento Experimental para a identificação das variáveis significativas para a resposta de Fenólicos totais (Y) $\left(\mathrm{mgEAG} \mathrm{L}^{-1}\right)$. 
Tabela 1 - Variáveis usadas no procedimento e respostas do planejamento.

\begin{tabular}{|c|c|c|c|c|}
\hline \multirow{2}{*}{ Amostra } & \multicolumn{3}{|c|}{ Variáveis } & Resposta \\
\cline { 2 - 5 } & $\mathrm{X}_{1}$ & $\mathrm{X}_{2}$ & $\mathrm{X}_{3}$ & $\mathrm{Y}$ \\
\cline { 2 - 5 } & Tempo (min) & $\begin{array}{c}\text { Razão chá- } \\
\text { solvente }\left(\mathrm{g} \mathrm{L}^{-1}\right)\end{array}$ & $\begin{array}{c}\text { Fração de Etanol } \\
(\%)\end{array}$ & $\begin{array}{c}\text { Concentração de } \\
\text { Fenólicos (mgEAG L }\end{array}$ \\
\hline \hline 1 & $-1(30)$ & $-1(10)$ & $-1(25)$ & $515,10 \pm 0,03$ \\
\hline 2 & $-1(30)$ & $0(55)$ & $1(75)$ & $1533,85 \pm 0,01$ \\
\hline 3 & $-1(30)$ & $1(100)$ & $0(50)$ & $4218,1 \pm 0,06$ \\
\hline 4 & $0(75)$ & $-1(10)$ & $1(75)$ & $537,99 \pm 0,10$ \\
\hline 5 & $0(75)$ & $0(55)$ & $0(50)$ & $2712,86 \pm 0,06$ \\
\hline 6 & $0(75)$ & $1(100)$ & $-1(25)$ & $4696 \pm 0,01$ \\
\hline 7 & $1(120)$ & $-1(10)$ & $0(50)$ & $497,93 \pm 0,05$ \\
\hline 8 & $1(120)$ & $0(55)$ & $-1(25)$ & $2821,6 \pm 0,03$ \\
\hline 9 & $1(120)$ & $1(100)$ & $1(75)$ & $3514,13 \pm 0,09$ \\
\hline
\end{tabular}

Os valores da Tabela 1 mostra que o maior valor de fenólicos totais é $4696 \mathrm{mgEAG} \mathrm{L}^{-1}$ correspondente ao tempo de $75 \mathrm{~min}$, razão chá-solvente $100 \mathrm{~g} \mathrm{~L}^{-1}$ e porcentagem etanol-água $25 \%$.

A partir das respostas da matriz do planejamento experimental, foi gerado um gráfico de pareto para verificar a significância do tempo, razão chá-solvente e fração de etanol na extração de polifenóis com solvente hidroalcóolico, representado na Figura 1.

Figura 1 - Gráfico de pareto para polifenóis para a representação das variáveis da extração hidoalcóolica.

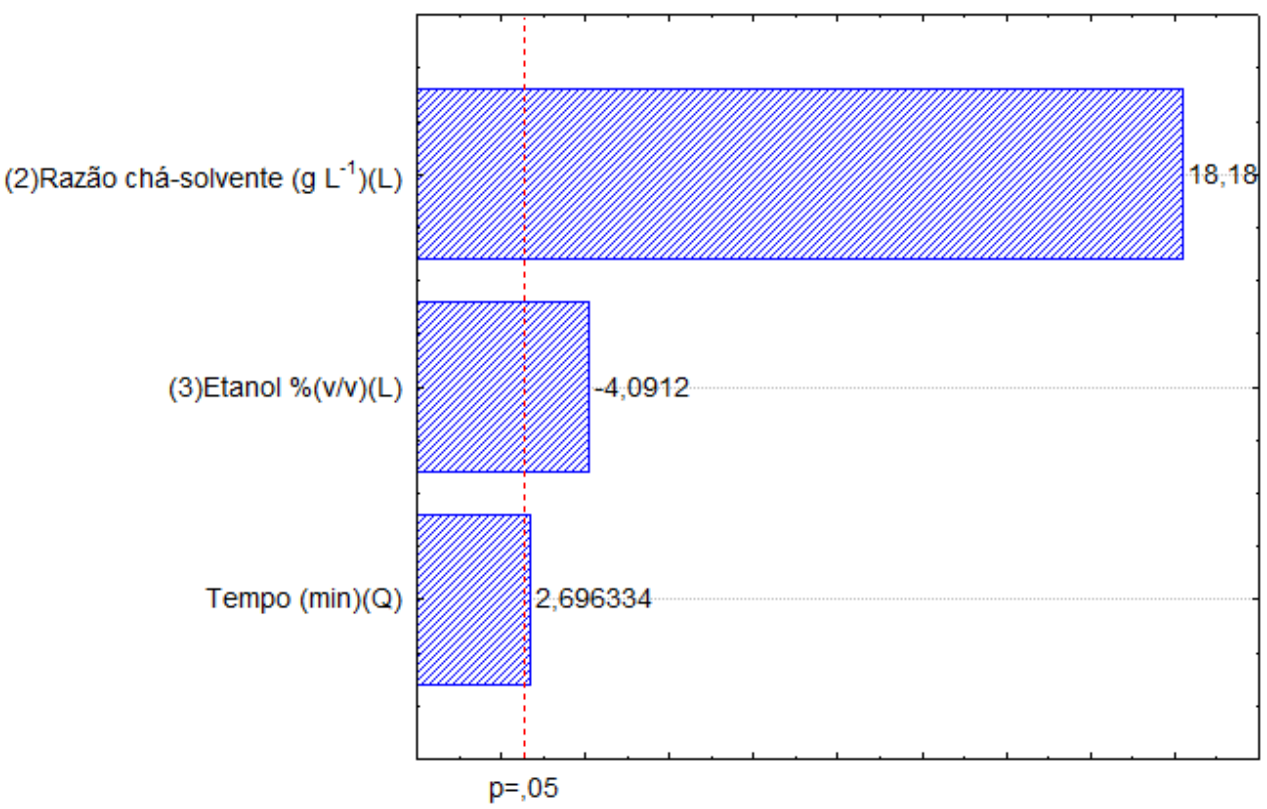

Como observado na Figura 1, as variáveis razão chá-solvente $\left(\mathrm{g} \mathrm{L}^{-1}\right)$ linear, fração etanol \%(v/v) linear e tempo (min) quadrático foram significativas $(\mathrm{p} \leq 0,05)$. A variável Razão chá-solvente $\left(\mathrm{g} \mathrm{L}^{-1}\right)$ linear tem efeito positivo e foi a de maior influência na extração de 


\section{Congresso Brasileiro de Engenharia Química em Iniciação Científica UFSCar - São Carlos - SP

polifenóis. Enquanto a variável fração de etanol \% (v/v) linear teve efeito negativo. A variável tempo (min) quadrático apresentou a menor influência na extração.

A equação 1 representa o modelo reduzido para resposta $\mathrm{Y}$ (polifenóis totais em $\mathrm{mg} \mathrm{L}^{-1}$ ) e o coeficiente de determinação $\left(\mathrm{R}^{2}\right)$.

$$
Y=2338,618+232,749 X_{1}{ }^{2}+1812,868 X_{2}-407,788 X_{3} \quad R^{2}=0,9861
$$

$\mathrm{O}$ valor do coeficiente de determinação $\mathrm{R}^{2}$ (equação 1 ) mostra que o modelo estatístico reduzido foi significativo para representar a extração de polifenóis do chá de hibisco. Desta forma, uma superfície de resposta baseada no modelo foi gerada a fim de representar os dados experimentais da extração de polifenóis em função das variáveis independentes significativas (tempo $(\mathrm{min})$, fração etanol \%(v/v) e razão chá-solvente $\left(\mathrm{g} \mathrm{L}^{-1}\right)$ ). As superfícies de resposta que representam a concentração de polifenóis em função de fração de etanol e tempo (Figura 2) e fração de etanol e razão chá-solvente (Figura 3) estão representadas a seguir.

Figura 2 - Superfície de resposta para extração de polifenóis $\left(m g E A G L^{-1}\right)$ em função da Fração de Etanol (\%) e Tempo (min).

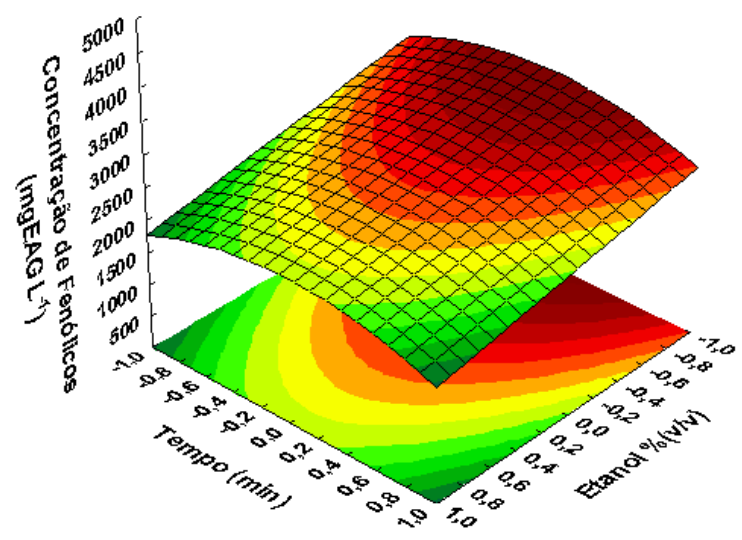

Figura 3 - Superfície de resposta para extração de polifenóis $\left(\mathrm{mgEAG} \mathrm{L}^{-1}\right)$ em função da Fração de Etanol (\%) e Razão chá-solvente $\left(\mathrm{g} \mathrm{L}^{-1}\right)$.

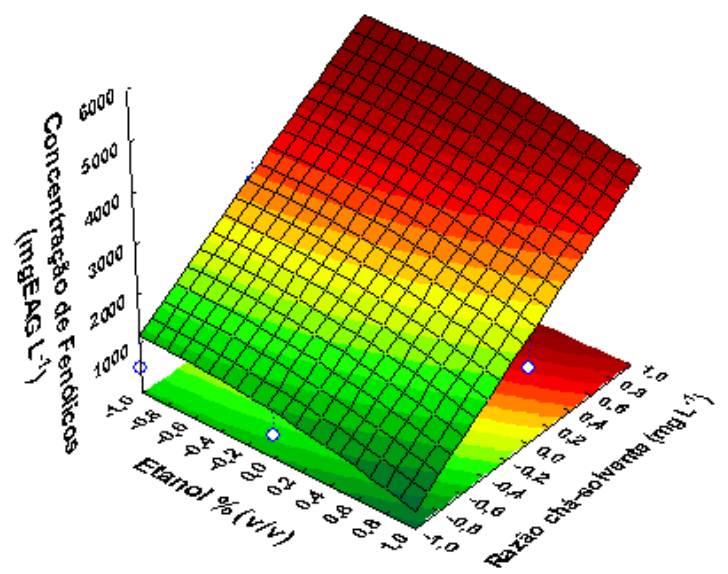


A partir da Figura 2, o valor mais adequado de polifenóis foi encontrado para a região de tempo no nível $0(75 \mathrm{~min})$ e fração de etanol $-1(25 \%)$, na Figura 3 o melhor valor encontrado foi para a região de nível de razão chá-solvente $1\left(100 \mathrm{~g} \mathrm{~L}^{-1}\right)$ e fração de etanol também -1(25\%), indicando que a condição desejada de extração foi com fração de etanol de $25 \%$, tempo $75 \mathrm{~min}$ e razão chá-solvente $100 \mathrm{~g} \mathrm{~L}^{-1}$.

No trabalho (Guindani et al.,2014) os autores utilizaram condições semelhantes de fração de etanol e razão chá-solvente e obtiveram uma concentração de compostos fenólicos de 3605,80( $\left.\mathrm{mgEAG} \mathrm{L}^{-1}\right)$, valor inferior à condição ótima de extração encontrada nesse neste trabalho 4696 (mgEAG L ${ }^{-1}$ ). (Vuong et al.,2011) estudaram os efeitos da razão chá-solvente na extração de tanino do chá verde e obtiveram o maior valor de tanino para razão chásolvente de $100 \mathrm{~g} \mathrm{~L}^{-1}$, a mesma relação encontrada nesse trabalho.

\section{CONCLUSÃO}

Com os resultados da extração dos compostos fenólicos do chá de hibisco foi possível constatar que misturas hidroalcóolicas se mostram eficientes na extração de polifenóis do Hibiscus. O maior valor de concentração de fenólicos totais 4696 (mgEAG L ${ }^{-1}$ ), foi obtido nas condições de solvente $25 \%$ de etanol em água, tempo de $75 \mathrm{~min}$ e razão chá-solvente 100 $\left(\mathrm{g} \mathrm{L}^{-1}\right)$.

\section{AGRADECIMENTOS}

Os autores agradecem à UFU (Universidade Federal de Uberlândia), a CAPES (Coordenação de Aperfeiçoamento de Pessoal de Nível Superior), à FAPEMIG (Fundação de Assistência à Pesquisa de Minas Gerais) e ao CNPq (Conselho Nacional de Desenvolvimento Cientifico e Tecnológico) pelo aporte financeiro.

\section{REFERÊNCIAS}

BORRÁS-LINARES, I. F.-A., S. ARRÁEZ-ROMAN, D. PALMEROS-SUÁREZ, P. A. DEL VAL-DÍAZ, R. ANDRADE-GONZÁLES, I. FERNÁNDEZ-GUTIÉRREZ, A. GÓMEZ-LEYVA, J. F. SEGURA-CARRETERO, A. Characterization of phenolic compounds, anthocyanidin, antioxidant and antimicrobial activity of 25 varieties of Mexican Roselle (Hibiscus sabdariffa). Industrial Crops and Products, v. 69, p. 385-394, 2015.

CHANG, H.-C. P., CHIUNG-HUEI YEH, DA-MING KAO, ERL-SHYH WANG, CHAUJONG. Hibiscus sabdariffa extract inhibits obesity and fat accumulation, and improves liver steatosis in humans. Food \& Function, v. 5, p. 734-739, 2014.

HU, C.-J. G., YING LIU, YANG ZHENG, XIN-QIANG YE, JIAN-HUI LIANG, YUERONG LU, JIAN-LIANG. Studies on the mechanism of efficient extraction of tea components by aqueous ethanol. Food Chemistry, v. 194, p. 312-318, 2016.

KUMAR, A. T., BARUN KUMAR DE, SIRSHENDU. Selective Extraction of (-)Epigallocatechin Gallate from Green Tea Leaves Using Two-Stage Infusion Coupled with Membrane Separation. Food and Bioprocess Technology, v. 5, p. 2568-2577, 2012. 
M. GUINDANI, F. T., F. KUHN, J. DAL MAGRO, F. DALCANTON ,M. A. FIORI, J. M. M. MELLO. Estudo do Processo de Extração dos Compostos Fenólicos e Antocianinas Totais do Hibiscus Sabdariffa U. C. d. R. d. Chapecó, 2014.

MAHADEVAN, N. S., KAMBOJ, P. Hibiscus sabdariffa linn.-An overview. Natural Product Radiance, v. 8, p. 77-83, 2009.

MELECCHI, M. I. S. Caracterização Química de Extratos de Hibiscus tiliaceus L: Estudo Comparativo de Métodos de Extração, Universidade Federal do Rio Grande do Sul, 2005.

MÔNICA JACHETTI MACIEL, M. P. P., HELOISA HELENA CHAVES CARVALHO, JOSÉ MARIA WIEST. Evaluation of the alcoholic extract of hibiscus (Hibiscus sabdariffa L.) as a protective antibacterial and antioxidant component. Inst Adolfo Lutz, 2012.

SILVA, A. P. G. V. A. D. S. G. C. D. Otimização da Extração de Compostos Fenólicos da Casca de Manga (Tommy Atkins) Utlizando Processo Assistido por Ultrassom. e-Xacta, 2016.

TOM VILLANI, H. R. J., JAMES E. SIMON, QING-LI WU. Hibiscus sabdariffa: Phytochemistry, Quality Control, and Health Properties. ACS Symposium Series, 2013.

VUONG, Q. V. S., COSTAS E. GOLDING, JOHN B. NGUYEN, MINH H. ROACH, PAUL D. Optimum conditions for the water extraction of $L$-theanine from green tea. Journal of Separation Science, v. 34, p. 2468-2474, 2011.

ZHEN, J. V., THOMAS S. GUO, YUE QI, YADONG CHIN, KIT PAN, MIN-HSIUNG HO, CHI-TANG SIMON, JAMES E. WU, QINGLI. Phytochemistry, antioxidant capacity, total phenolic content and anti-inflammatory activity of Hibiscus sabdariffa leaves. Food Chemistry, v. 190, p. 673-680, 2016. 